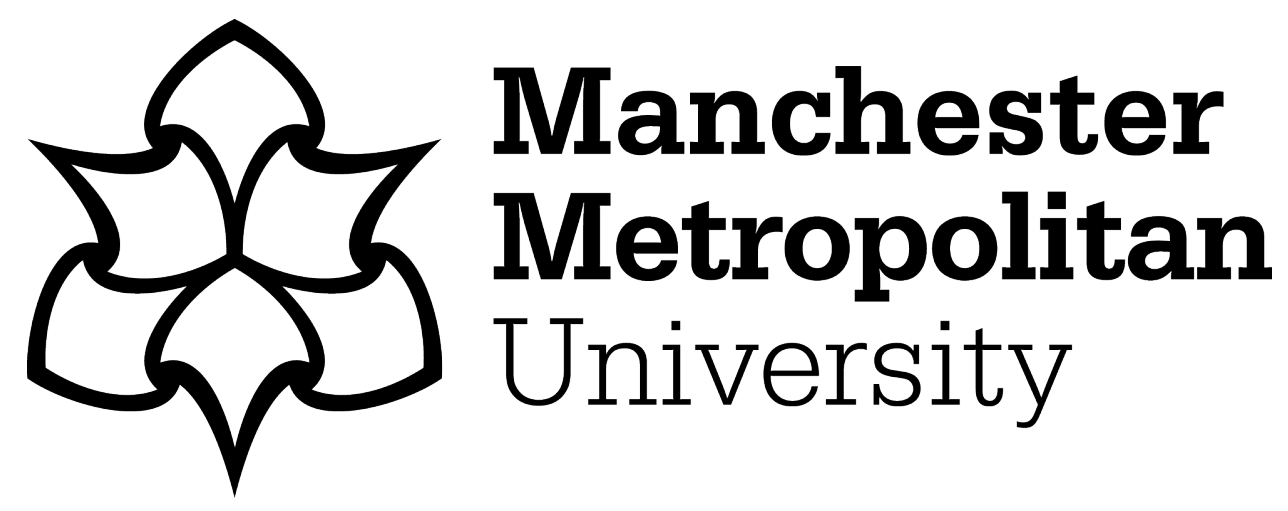

Rowley, Jenny ORCID logoORCID: https://orcid.org/0000-0003-3437-6914 and Keegan, Brendan ORCID logoORCID: https://orcid.org/0000-00028441-3763 (2020) An overview of systematic literature reviews in social media marketing. Journal of Information Science, 46 (6). pp. 725-738. ISSN 0165-5515

Downloaded from: https://e-space.mmu.ac.uk/623403/

Version: Accepted Version

Publisher: SAGE Publications

DOI: https://doi.org/10.1177/0165551519866544

Please cite the published version 


\title{
An overview of systematic literature reviews in social media marketing
}

\begin{abstract}
Systematic literature reviews (SLRs) adopt a specified and transparent approach, in order to scope the literature in a field or sub-field. However, there has been little critical comment on their purpose and processes in practice. By undertaking an overview of SLRs in the field of social media (SM) marketing, this article undertakes a critical evaluation of the SLR purposes and processes in a set of recent SLRs and presents a future research agenda for social media marketing. The overview shows that the purposes of SLRs include: making sense (of research in a field), developing a concept matrix/taxonomy, and supporting research and practice. On SLR processes, whilst there is some consensus on the stages of the process, there is considerable variation in how these processes are executed. This article offers a resource to inform practice and acts as a platform for further critical debate regarding the nature and value of SLRs.
\end{abstract}

\section{Keywords}

Literature review; social media marketing; social media user behaviour; systematic literature review 


\section{Introduction}

Literature reviews are a familiar aspect of the research process. In management research, literature reviews are used to manage the diversity of knowledge as a prelude to a specific enquiry [1], or more broadly to integrate a body of scholarship [2]. For example, the type of literature review that is typically part of every research article, referred to in this article as a narrative literature review, has a number of functions. These include: supporting the identification of research topics, questions or hypotheses; identifying the literature to which the research will make a contribution, and contextualizing the research within that literature; building an understanding of theoretical concepts and terminology; facilitating the building of a bibliography or list of the sources that have been consulted; suggesting potentially useful research methods; and, analysing and interpreting results [3]. The process associated with the creation of these reviews typically has three stages: initial review (at the beginning of the research process); ongoing monitoring of the literature as the research topic and processes evolve; and re-visiting the literature in the writing-up phase. Although there are recognised search strategies associated with such reviews, such as citation pearl growing, Briefsearch, building blocks, and successive fractions [3], the detail of the processes that lead to narrative literature reviews, including for example, selection and evaluation criteria are rarely reported [4] and the criteria for inclusion are typically qualitative, based on the reviewers' judgement [2]. However, Aguinis, Ramani and Alabduljader [5] suggest that there is a need for transparency at each stage of the research process, including the development of the theoretical base for the research.

Less familiar than narrative reviews, but being increasingly recognised for their value, are SLRs, sometimes referred to as systematic reviews of literature. Such reviews differ from narrative literature reviews in both purpose and process. Cook et al [6] suggest that 'systematic reviews differ from traditional narrative reviews by adopting a replicable, 
scientific and transparent process... that aims to minimize bias through exhaustive literature searches of published and unpublished studies and by providing an audit trail of the reviewers' decisions, procedures and conclusions' (p.378). Littel, Corcoran and Pillai [7] suggest that SLRs 'comprehensively locate and synthesize research that bears on a particular question, using organized, transparent, and replicable procedures' (p.1). Importantly, SLRs are seen to offer a contribution to knowledge in their own right, and whilst they can be presented as part of a research article reporting empirical research, they are often published as a separate article. Their purpose is to identify key contributions in a field, and to identify patterns in the knowledge base, using a transparent process, with a view to informing future research and practice guidelines $[8,9]$. Typically, this involves profiling the research on a specified topic in terms of its bibliographic characteristics (e.g. time span, key journals), and developing a concept matrix [10] of key themes to aid in summarizing the current state-of-the-art relating to the research question or topic, and proposing a future research agenda. In terms of process, SLRs are characterised by their adoption of a specified replicable, scientific and transparent process to the identification of articles in a field, the refinement of any initial dataset, and the sense-making associated with the development of a narrative associated with the field $[11,2]$. In other words, SLRs typically have a 'methodology' section, and hence can be regarded as literature-based research projects offering a contribution to knowledge in their own right.

To add to the complexity, various authors have proposed a number of different types of SLRs $[2,12]$. The most important distinction for the purpose of this article is that between qualitative and quantitative SLRs. At the heart of a quantitative SLR typically lies an analysis and integration of the quantitative data reported in the SLR, whereas for qualitative SLRs the review process involves a more iterative surfacing of research questions, searching and protocol development [12]. The qualitative process model has most resonance with the 
development of SLRs in computing, information science and management, due to the diversity of research methods and approaches adopted in these fields.

Despite the increasing numbers of SLRs being published, no comparative or critical evaluation has been conducted into the practice associated with SLRs in computing and information science or business and management. Yet, Fisch and Block [13] suggest that despite widely available guidance on conducting systematic reviews, there is considerable variability in the quality of systematic reviews in management science, and Boell and CecezKecmanovic [14] raise concerns regarding the potential impact of SLRs on critical engagement with the information systems literature. Hence, the research question at the heart of this article is: to what extent is the practice in SLRs consistent, and consistent with good practice guidelines? The aim of this paper is to undertake a critical and comparative analysis of the purpose and processes associated with SLRs, in practice, through examining recent SLRs in the social media marketing field. The research concludes with a critical comparison and distillation of the research agendas proposed in the selected reviews; this process generates a meta-agenda for future research in social media marketing.

\section{Literature Review}

This section draws together prior commentary on literature reviews, with a focus on SLRs and their definitions, purposes, and processes

\subsection{Definitions}

In recent years, there have been two major drivers for interest in, and increased use, of SLRs: concern regarding the quality of traditional narrative literature reviews, and the increased focus on underpinning research and practice with a strong evidence base. Denyer and Tranfield [15] suggest that 'a systematic review is a specific methodology that locates existing studies, selects and evaluates contributions, analyses and synthesize data, and 
reports on the evidence in such a way that allows reasonably clear conclusions to be reached about what is known and what is not known' (p.671). An SLR uses a process that is 'replicable, transparent, objective, unbiased and rigorous' ([14] p.121).

\subsection{Purpose of SLRs}

Both narrative and SLRs enable the researcher to manage the diversity of knowledge in a specific academic enquiry. They help the researcher to both map and assess the existing intellectual territory, and to specify a research question, which will advance existing knowledge or theory [11]. Torraco [16] summarises the purpose of literature reviews, thus: 'the ...literature review is a form of research that reviews, critiques, and synthesizes representative literature on a topic...such that new frameworks and perspectives on the topics are generated' (p.356). Webster and Watson [8] suggest that narrative reviews: create a firm foundation for advancing knowledge; facilitate theory development; close areas where a plethora of research exists; and, uncover areas where research is needed.

The functions discussed above are relevant for both narrative and systematic reviews. However, systematic reviews differ from narrative reviews in a number of respects. In response to criticisms regarding the lack of transparency regarding the processes associated with narrative literature reviews, various authors have promoted the value of SLRs $[15,17]$. In addition, systematic reviews are often wider in scope that narrative reviews, often centring on a research topic or field, in contrast to narrative reviews, that typically focus on a more specific research question. SLRs have greater potential to increase the theoretical coherence of a field. As such, they are particularly beneficial in fields such as organization and management studies, and computing and information science, which have a large and fragmented research base [18]. If this research base is, like social media marketing, interdisciplinary and rapidly evolving, the value of an SLR is further enhanced. Finally, 
systematic reviews can make research findings more accessible to practice, and thereby have the potential to drive increased rigor of practice. For example, in medicine, SLRs are widely used to promote evidence-based practice [11]. The PRISMA statement, the result of international collaboration between medical and health sciences researchers, and subject to ongoing revision, offers a 27 -item checklist for reporting a systematic review, covering title, abstract, introduction, methods, results, discussion, and funding [9]. No such list exists in management and information sciences, nor is there any significant evidence to suggest that SLRs inform practice. A number of barriers hinder progress in these disciplines, including: concerns regarding the quality of SLRs; the need to accommodate qualitative (as well as quantitative) evidence to create actionable knowledge; the heterogeneity of research methodologies; and, the widely recognized and long-standing gulf between research and practice [15].

\subsection{Processes}

Table 1 summarises the main processes in conducting a literature review. Webster and Watson [8] and Torraco [16] discuss narrative reviews, whilst Tranfield et al [11] and Fisch and Block [13] propose models for the SLR process. Whilst all of the four models have some similarities, it is very evident that they are not identical. Firstly, and most obviously, the models differ in the number of distinct steps in the SLR process. Secondly, there are steps that occur in some reviews, but not in others. All authors agree that the start of the process is an identification of the research topic, although Fisch and Bock [13] also suggest the identification of a research question. Some authors then suggest the need to justify the need for a literature review $[11,16]$. Tranfield et al [11] more specifically identify the process in the proposal stage of the SLR, than do other authors. Not surprisingly, all authors are agreed that a key stage is the 'review of prior literature' [8], 'search and retrieve the appropriate literature(s)' [16], or 'identify the relevant literature in a systematic way' [13]. However, 
Tranfield et al [11] articulate the processes in the stage of conducting a review in more detail than do other authors. In particular, they suggest the steps: study quality assessment, data extraction, and monitoring progress. The evaluation of the study quality is of particular significance. Next, only Webster and Watson [8] suggest developing a model to guide future research, whilst Torraco [16] and Tranfield et al [11] point to the need for synthesis. Webster and Watson [8], together with Tranfield et al [11], also comment on the need to consider the implications for practice. Researchers are likely to be strongly influenced by the steps outlined in models such as these. However, the inconsistencies between these models suggest that there is scope for further work on the articulation of the stages within the SLR process. A lack of consensus regarding the steps in the SLR process amongst some of the lead commentators has serious potential to undermine consistency in SLR practice.

Table 1. Processes of SLRs identified by key prior works on SLRs

\begin{tabular}{|c|c|}
\hline Author (s) & Processes \\
\hline $\begin{array}{l}\text { Webster and } \\
\text { Watson [8] }\end{array}$ & $\begin{array}{l}\text { 1. Motivate the research topic } \\
\text { 2. Describe the key concepts } \\
\text { 3. Delineate the boundaries of the research } \\
\text { 4. Review relevant prior literature in the core and related areas } \\
\text { 5. Develop a model to guide future research } \\
\text { 6. Justify propositions, by presenting theoretical explanations, past } \\
\text { empirical findings and practical examples } \\
\text { 7. Present implications for researchers and managers }\end{array}$ \\
\hline Torraco [16] & $\begin{array}{l}\text { 1. Identify an appropriate topic or issue for the review } \\
\text { 2. Justify why a literature review is the appropriate means of } \\
\text { addressing the topic or problem } \\
\text { 3. Search and retrieve the appropriate literature(s) } \\
\text { 4. Analyse and critique the literature } \\
\text { 5. Create new understandings of the topic through one or more forms } \\
\text { of synthesis }\end{array}$ \\
\hline $\begin{array}{l}\text { Tranfield et al. } \\
\text { [11] }\end{array}$ & $\begin{array}{l}\text { Stage 1: Planning the Review } \\
\text { Identification of a need for a review } \\
\text { Preparation of a proposal for a review } \\
\text { Development of a review proposal } \\
\text { Stage II: Conducting a Review }\end{array}$ \\
\hline
\end{tabular}




\begin{tabular}{|c|c|}
\hline & $\begin{array}{l}\text { Identification of research } \\
\text { Selection of studies } \\
\text { Study quality assessment } \\
\text { Data extraction and monitoring progress } \\
\text { Data synthesis } \\
\text { Stage III: Reporting and dissemination } \\
\text { The Report and recommendations } \\
\text { Getting evidence into practice. }\end{array}$ \\
\hline $\begin{array}{l}\text { Fisch and Block } \\
{[13]}\end{array}$ & $\begin{array}{l}\text { 1. Motivate the topic and state the research question } \\
\text { 2. Identify the relevant literature in a systematic way } \\
\text { 3. Choose the right balance between breadth and depth } \\
\text { 4. Focus on concepts, not studies } \\
\text { 5. Derive meaningful conclusions } \\
\text { 6. Follow a coherent article structure }\end{array}$ \\
\hline
\end{tabular}

\subsection{Field of Study: Social Media Marketing}

This article focusses on literature reviews in the area of social media marketing. For the purposes of this article, the topic is taken to cover all aspects of the use of social media by businesses and other organizations to communicate with, or gather data regarding, their customers and other stakeholders. This study also includes research articles relating to customer or user behaviour in organizational social media spaces. This definition of social media marketing is further operationalized through the search terms and protocol outlined in the next section.

Social media marketing was chosen as the topic for this study partly because the opportunity presented itself. The authors of this article are social media marketing researchers, and on their research journey encountered a number of SLRs on closely related, but distinct aspects of social media marketing. Being researchers in social media, with complementary expertise in information science, who have also published SLRs, the authors had the expertise to undertake an analysis of these documents. In addition, social media marketing is a field that is undergoing significant growth in both research and practice [19]. Denyer et al [18] suggest 
that significant growth in a field can lead to fragmentation of the knowledge base. In the case of social media marketing, the risk of such fragmentation is heightened by the interdisciplinary nature of the field. Further, growth and developments in research are paralleled by an even greater rate of change in in social media marketing technologies, strategies, practice, and user behaviours. In such a context, evidence-based practice is very important, but also very difficult [20]. Finally, as Webster and Watson [8] suggest progress in interdisciplinary fields, such as information systems can be complex because such fields, especially in their infancy, need to draw on theories from a variety of other fields. SLRs have an important role in accelerating the accumulation of knowledge in the field and building theoretical foundations for the field. On the other hand, Torraco [16] points out that literature reviews addressing new and emerging topics (such as social media marketing) are more likely to lead to an initial or preliminary conceptualization of the topic (i.e. a new model or framework) rather than a reconceptualization of previous models. Hence, an examination of SLR practices in the field of social media marketing has potential to develop insights and guidelines for other nascent and inter-disciplinary areas.

\section{Methodology}

The aim of this article is to perform a meta-analytic review of social media marketing SLRs. The much-cited advice from Webster and Watson [8] suggests that SLRs in social sciences should adopt a clearly identifiable and accountable process, which significantly advances the field by offering a point of view about the phenomenon under scrutiny. This study, therefore reports on the methodology used in this meta-analytic review. Where appropriate, it adopts and adapts the processes normally associated with SLRs $[11,21]$.

Step 1 involved the identification of an aim for the study that also specified its scope, 
$v i z$ : to undertake an analysis of recent SLR research articles that have examined the social media marketing field. Step 2 focussed on the selection of appropriate databases for the search. Business Source Premier was selected as the vehicle for the literature search process. Step 3, which involved the selection of key word strings, was followed by Step 4, searching using the keyword strings. Initial searching was conducted using the primary keyword string: 'systematic literature review OR systematic review of the literature AND social media OR social networking'. This initial search led to the identification of three additional strings: value creation, social commerce, and online communities. These were used to conduct a further phase of searching. Search on both the initial and additional search strings led to a database of 42 results.

Step 5 focussed on the refinement of the dataset using exclusion criteria. The primary criterion for exclusion was that the source should be an SLR on social media marketing. The application of this criteria led to the exclusion of 20 of the retrieved SLRs. Typical of the SLRs that were excluded was one on social media in the field of psychology. A second criterion was that the article should present a clear methodology. For example, a number of articles within the management discipline, whilst purporting to be systematic reviews, offered no clear methodology, and, in actuality, employed a narrative style of literature review. The third criterion was that SLR's need to be peer-reviewed and published in academic journals; using this criterion, 7 conference papers were excluded. Next, sources were excluded based on quality. To ensure rigour of the meta-analytic review, non-peerreviewed SLRs were excluded, which included 6 conference papers. The final dataset comprised 13 SLRs.

Step 6 of the review process focussed on analysis of the SLRs. To perform the metaanalytic review, a bibliographic review of the dataset was conducted to identify patterns and dissimilarities between the approaches adopted in the reviews, with regard to the three 
characteristics of the reviews: purpose, process, and further research agendas. As the analysis proceeded, thematic categorisations emerged for each of these three characteristics, as summarised in Tables 2 and 3 and in the headings in the process section of the Findings. The articles selected for inclusion are shown in the top rows of tables 2 and 4.

\section{Findings}

\subsection{Purpose of SLRs}

The most commonly mentioned purposes are: making sense, developing a concept matrix or taxonomy, and helping researchers and practitioners. For example, Lamberton and Stephen [22] suggest: 'Our contribution lies in helping researchers... and... developing new research directions to advance the literature and offer relevant insights for marketing practice' (p.147), whereas Effing and Spil[23] aim to 'develop a framework for the analysis of social media strategies' (p.1). Busalim et al [24] seek to 'explore the social commerce concept'... and [thereby] to provide a mind map of the s-commerce themes for researchers who want to recognise the topic areas where more research is needed...[and] for practitioners, [their] review brings them up to date on the s-commerce activities and the current state of s-commerce and its historical development' (p.1076). Salo [25] performed their SLR to examine how the deployment of social media influences organizational decision-making in B2B environment.

On audiences, Baethge et al [26] seek to inform not only researchers and practitioners, but also editors, and reviewers, by providing them with an account of the current state-ofthe art. In their review and synthesis of the literature on online word-of-mouth (eWOM), King et al [27] refer to examining 'what we know' and posing critical research questions as a guide to 'what we need to know'. 
Other authors seek to draw together a fragmented research literature. For example, Ketonen-Oksi et al [28], in their review of social media-based value creation, seek to make sense of a 'research literature ... [that is] still fragmented into case studies of various microlevel study contexts' (p.2). Zhang and Benyoucef [29] suggest that in respect of consumer behaviour in social commerce, 'current research is rather fragmented, which makes it difficult to derive meaningful and conclusive implications' (p.95). In the context of eWOM, King et al [27] also highlight that fragmentation may also be encountered as a result of the use of different research methods. Ngai et al [30] take a slightly different perspective, using a SLR to 'understand the causal relationships among different research constructs' (p.34) and identifying theories, conceptual models and frameworks used in social media research. 
Table 2. SLR Purposes

\begin{tabular}{|c|c|c|c|c|c|c|c|c|c|c|c|c|c|c|}
\hline & 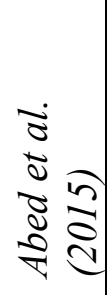 & 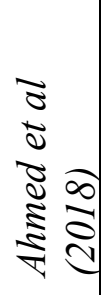 & 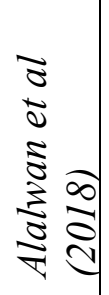 & 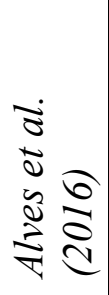 & 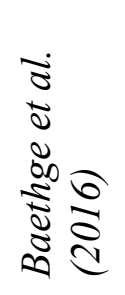 & 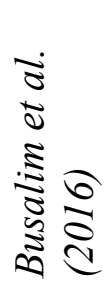 & 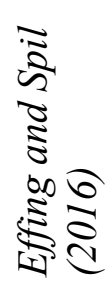 & 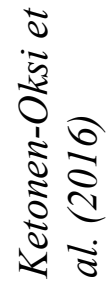 & 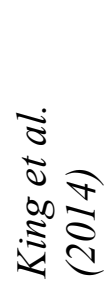 & 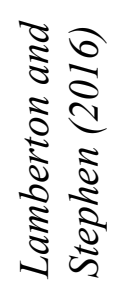 & 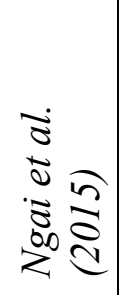 & 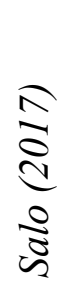 & 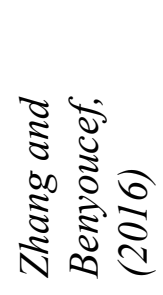 & $\tilde{\Xi}$ \\
\hline Making sense & & & & $\mathbf{X}$ & $\mathbf{X}$ & $\mathbf{X}$ & $\mathbf{X}$ & & $\mathbf{X}$ & $\mathbf{X}$ & $\mathbf{X}$ & & $\mathbf{X}$ & 8 \\
\hline Research agenda & & $\mathbf{X}$ & & & $\mathbf{X}$ & & & & & $\mathbf{X}$ & $\mathbf{X}$ & $\mathbf{X}$ & & 3 \\
\hline Synthesize perspectives & & $\mathbf{X}$ & $\mathbf{X}$ & $\mathbf{X}$ & & $\mathbf{X}$ & & $\mathbf{X}$ & $\mathbf{X}$ & $\mathbf{X}$ & & & $\mathbf{X}$ & 6 \\
\hline $\begin{array}{l}\text { Understand evolution } \\
\text { of a research domain }\end{array}$ & & & & & $\mathbf{X}$ & $\mathbf{X}$ & & & $\mathbf{X}$ & $\mathbf{X}$ & $\mathbf{X}$ & & $\mathbf{X}$ & 6 \\
\hline $\begin{array}{l}\text { Develop a concept } \\
\text { matrix/taxonomy }\end{array}$ & $\mathbf{X}$ & & & $\mathbf{X}$ & $\mathbf{X}$ & $\mathbf{X}$ & & $\mathbf{X}$ & $\mathbf{X}$ & $\mathbf{X}$ & $\mathbf{X}$ & & & 8 \\
\hline Establish rigour & & & & & $\mathbf{X}$ & $\mathbf{X}$ & & & & $\mathbf{X}$ & & & $\mathbf{X}$ & 4 \\
\hline $\begin{array}{l}\text { Draw together a } \\
\text { fragmented literature } \\
\text { base }\end{array}$ & & & & $\mathbf{X}$ & & & $\mathbf{X}$ & $\mathbf{X}$ & $\mathbf{X}$ & & $\mathbf{X}$ & & & 5 \\
\hline $\begin{array}{l}\text { Identify theoretical } \\
\text { perspectives }\end{array}$ & & $\mathbf{X}$ & & $\mathbf{X}$ & & $\mathbf{X}$ & $\mathbf{X}$ & & & $\mathbf{X}$ & $\mathbf{X}$ & $\mathbf{X}$ & $\mathbf{X}$ & 6 \\
\hline $\begin{array}{l}\text { Help researchers and } \\
\text { practitioners }\end{array}$ & $\mathbf{X}$ & & & & & $\mathbf{X}$ & $\mathbf{X}$ & $\mathbf{X}$ & $\mathbf{X}$ & $\mathbf{X}$ & $\mathbf{X}$ & & $\mathbf{X}$ & 8 \\
\hline Identify key issues & $\mathbf{X}$ & & & $\mathbf{X}$ & & $\mathbf{X}$ & $\mathbf{X}$ & $\mathbf{X}$ & $\mathbf{X}$ & $\mathbf{X}$ & & & & 7 \\
\hline
\end{tabular}




\subsection{Process}

On the basis of a review of the phases and their processes adopted by the authors of the SLRs in our dataset, six phases were identified: formulating research objectives and questions; developing search strategies and identifying relevant documents; formulating and applying exclusion criteria; bibliographic analysis of the final dataset; development of a concept matrix and thematic analysis; theory analyses, and the development of a future research agenda. Some of these clearly parallel the purposes of an SLR as listed in Table 1. Some SLRs also included other phases such as selecting research questions and applying methodological screening criteria [28]. More details on the processes and approaches adopted with regard to each of these processes are elaborated below.

Table 3. SLR Process Phases

Formulating research objectives and questions

Developing search strategies and identifying relevant documents

Formulating and applying exclusion criteria

Bibliographic analysis of the final dataset

Development of a concept matrix and thematic analysis

Development of a future research agenda

Formulating research objectives and questions. Most SLRs identify specific aims and objectives and/or research questions. For example, Effing and Spil [23] identify their objective as being 'to discover key elements of social media strategies and review existing frameworks, methods, theories and standards for the development of social media strategies' 
(p.2), whilst Busalim et al [24]'s objective is to 'explore the s-commerce' concept. Ahmed et al [31] have three objectives: (i) to collect, summarize, analyse and synthesize information regarding the accuracy and values of previous literature, (ii) to report on the empirical findings of this domain's existing studies, and to (iii) identify knowledge gaps for future research. Salo [25], on the other hand, simply poses the research question: 'How much research exists on the social media deployment in the industrial marketing area, and what are the possible future research directions for industrial marketing?' (p. 115).

Good practice suggests that in undertaking a literature review, research questions and objectives should be underpinned by a theoretical stance, or at least, a specification of the discipline to which the review seeks to contribute. The disciplines to which the SLRs included in this article seek to contribute is implicit in the journal in which they have been published. On this basis, three clear groups are in evidence: 'marketing' (four reviews), 'information management' (seven reviews), and 'marketing and information management' (two reviews). Most reviews do not explicitly mention a theoretical stance. Exceptions are Ketonen-Oksi et al [28], who refer to service-dominant logic, and Zhang and Benyoucef [29] who refer to the stimulus-organism-response model. On the other hand, Lamberton and Stephen [22] and Ngai et al. [30] do report on the diverse range of theories in evidence in the articles included in their SLR. This raises some questions regarding how the issue of theoretical stances can and should be tackled in the context of interdisciplinary fields such as social media marketing.

Developing search strategies and identifying relevant documents. Virtually all of the articles in the dataset identified the timeframe over which a search was conducted. For example, Lamberton and Stephen [22] examined articles published between 2000 and 2015, whilst Baethge et al [26], after a careful assessment of the timeline associated with social commerce searched between 2007 and 2014, and Ahmed et al [31] literature base was 
between 2010 and 2016. On the other hand, Effing and Spil [23] do not identify any specific timespan. Timelines can also be useful in mapping the evolution of a research field [24].

There is a very strong consensus, consistent with the authoritative guidelines on conducting SLRs [17] that there is a need to specify search strategies in terms of sources used and search strategies, although the approaches used, and the details provided vary considerably. In terms of sources, most authors used a selection on the following databases: EBSCOhost, Google Scholar, ScienceDirect, Social Sciences Citation Index, Scopus, Web of Science, ABI- Inform, Business Source Premier, Science Direct, Emerald, Wiley Online Library. This wide range of databases is reflective of the inter-disciplinary nature of social media marketing and the need to search across literature in several disciplines [27].

Some authors either supplemented or substituted their search of major databases with direct searching of highly regarded or ranked journals in the field [26, 29], or through following-up citations of the articles identified through the search process in the databases [24], otherwise referred to as snowball searching [22]. Furthermore, both Busalim et al [24] and Ahmed et al [31] made use of Mendeley to eliminate duplicates.

Search strategies were developed based on key words. Some authors report on a list of typical keywords (e.g. [27]), whereas others, such as Baethge et al [26] provide a detailed analysis of the iterative development of their set of keywords and search strategies through identifying initial articles. Most authors report on the development of synonym and word variant lists e.g. social media, Web 2.0, enterprise 2.0. [28]. Interestingly, Lamberton and Stephen [22], when extending their search outside of the academic literature to white papers and industry reports, converted their 'academic search terms into keywords that were more likely to appear in the business press' (p.148). Finally, several authors offer some kind of report on the results from their various research strategies $[22,23,25,26]$. 
Formulating and applying exclusion criteria Most authors used exclusion criteria. Most common amongst these were: book chapters, reports and conference proceedings ' $d u e$ to the variability in their respective peer review processes and more restricted availability' [32, p.1030]. Refereed journal articles were regarded as representing 'state-of-the-art research outputs' [30, p.34]. Some reviews included only empirical articles (e.g. [30]) or even more restrictively, articles with a defined sample and empirical methodology [27]. Inclusion criteria typically involve: full-text available, published within the selected period of time, relevant to the topic of the review, listed in the selected databases, and written in English [24, 28]. Duplicates are eliminated from the dataset. Finally, authors of most SLRs will need to manually analyse each article, examining its title, abstract and, possibly, full text to determine its suitability for inclusion in the final dataset $[23,26]$, although several of the articles did not report on this stage. It is usual at this stage for authors to report the number of articles remaining in the dataset and being taken forward for further analysis in the next two stages.

Bibliographic analysis of the final dataset Bibliographic analyses of the final dataset are often included to offer a profile of the selected articles. These may show: the number of articles published annually over the period of the reviews [22, 23, 24, 29, 31, 33]; research strategies and methods [19, 23, 24, 28, 29, 32]; and, theoretical foundations [24,29, 30].

In some instances, this is coupled with the identification of key themes (see below), to support the analysis of the profile of the dataset, not just as a whole, but with references to specific themes or topics. For example, Lamberton and Stephen [22] use this approach to identify three eras of digital, social and mobile marketing, within which they discuss progress under a number of themes, whilst Baethge et al [26] couple reporting on date with reporting on disciplines (marketing, electronic commerce, IS conference and IS journals). In addition, some studies (e.g. [32]) conduct a count by theme, to support a subsequent discussion of the 
literature on a specific theme.

Development of a concept matrix and thematic analysis A key step towards identifying the topics that might merit further attention is the development of a concept matrix of the key themes covered by the literature in the dataset. This enables the identification of important research questions in the field, and an assessment of which questions would benefit from further research. Different authors used different approaches to develop and apply this concept matrix. Lamberton and Stephen [22], for example, used a combination of date of publication and key word analysis, to identify three eras and the key research topics within those eras. The last of these eras they labelled 'the age of social media' and discussed the following themes within this era: individual self-expression as a means of amplifying or dulling marketing actions, user-generated content as a marketing tool, capturing marketing intelligence in specific social media platform. In developing their research themes, Baethge et al [26] analysed and adapted categories of research themes proposed by other authors writing on social commerce. Their categories were: user behaviour, website design, enterprise strategies, social process, adoption strategy, business model, security and privacy policies, network structure, firm performance, and overview. It is unclear how Busalim et al [24] surfaced their taxonomy of research themes on social commerce, but they more or less replicate those used by Baethge et al [26]. Similarly, it is unclear how Alves et al [32] surfaced their key themes, but they present two main groups of themes, firm/organisation and consumer, and sub-themes within these areas. For firm/organization: degree of use and facility of using SM; optimisation, measurement, and impact of social media marketing strategies; abusive/unethical use. For consumer: increased consumption; use, search, and a share of information; attitude toward the brand; and influence among consumers. Similarly, although, Ahmed et al [31], Salo [25] and Alawan et al [19] generated a list of themes, they offer no explicit explanation of the process whereby 
the themes were adopted. Zhang and Beyouncef [29] adopt a rather different approach in their review of consumer behaviour in social commerce. Their analysis is informed by the stimulus-organism-response model of human behaviour, coupled with the five-stage consumer decision-making process (need recognition, search, evaluation, purchase, and post-purchase). These theories are used to distil the literature and to propose a 'complete theoretical framework for consumer behaviour in social commerce'. King et al [27] also use a previous theoretical framework to help to structure both their review of the previous literature and their proposals for future research in four quadrants associated with antecedents of eWOM Senders, consequences for eWOM senders, the antecedents of the receiver, and the consequences to the receiver. Adopting yet another variation, Ngai et al [30], with an interest in causality between research constructs in social media research, proposes some categories of attributes, each with sub-categories. These categories are: antecedents, mediators, moderators, and outcomes; they are used as the basis of a causal chain framework for social media research.

Development of a future research agenda Most articles suggest an agenda for future research on the basis of their literature review. As Baethge et al [26] assert, 'by providing a research agenda, we hope that our results will stimulate and guide future research in this exciting field' (p.1). Some researchers use the themes in the concept matrix to analyse and report on past research, and to assist in framing a future research agenda. For example, King et al [27], as described above, offer an elaborate research agenda, which embraces eleven specific research questions. Busalim et al [24] offer a simple research agenda for scommerce, with three key themes: theoretical considerations, customer behaviour considerations, and design and implementation considerations, each with three or four related research questions. Salo [25] delineates four areas for the future research of social media in an industrial marketing context: influence, supplier customer acquisition and 
customers, business relationship and networks, business outcomes, and competition. Other authors offer a narrative account, without the identification of specific themes. For example, Alves et al [32] offers a narrative account that identifies a number of future lines of research, embracing both consumer and firm behaviours, whilst Ngai et al [30] offer a narrative discussion of future research directions, organized under the following four headings: organisation orientation, social power, cultural differences and impacts of social media. In contrast, some studies are less prescriptive; they do not specifically propose an agenda for further research, but rather suggest that their theoretical framework may inspire related research in the future $[19,29,31]$.

Identification of limitations of the reviews. Only a few authors include any statement on the limitations of their reviews. Three of these $([26,29,30])$ suggest that their review could be improved by widening the scope of their study, to include either topics or types of publications that were excluded on the basis of the specified criteria of their study. Other suggest that the quality of their review was affected by the variable quality of the underlying studies [28]. Aalawan et al. [19] argues the case for a meta-analysis of the datasets generated by the various studies, and further evaluation of the main research methods used in studies in this field. Finally, Salo [25] points to a number of areas where further attention would enhance systematic reviews: selection of articles, the identification of thematic categories, and the formulation of future research agendas.

\subsection{Towards a research agenda for social media marketing}

This section conducts an overview of the SLRs in social media marketing analysed in this article. Table 4 identifies six themes in this research agenda and indicates the extent to which each of these is covered by the different SLRs. The theme, social media practice and strategy is included in all of the reviews. Other widely covered themes are social media user 
behaviour, and social media research approaches. On the other hand, only three SLRs cite the important topic of social media privacy and security as an area for further research. More specific details on the topics mentioned under each of the themes are discussed below. 
Table 4. Agendas for future research

\begin{tabular}{|c|c|c|c|c|c|c|c|c|c|c|c|c|c|c|}
\hline & $\begin{array}{l}\vec{\Xi} \\
\tilde{n} \\
\vec{\sigma} \\
\tilde{\sigma} \\
\tilde{\Xi} \\
\tilde{\nabla}\end{array}$ & 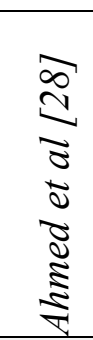 & 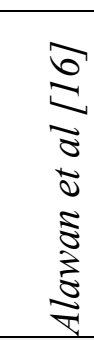 & 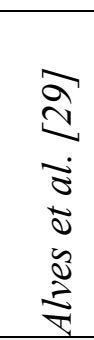 & 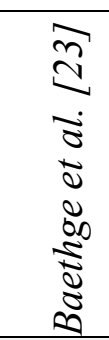 & 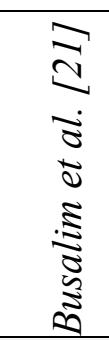 & 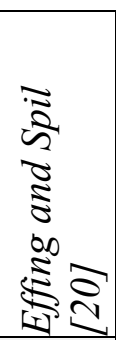 & 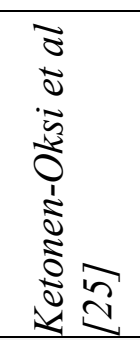 & 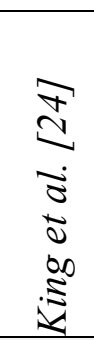 & 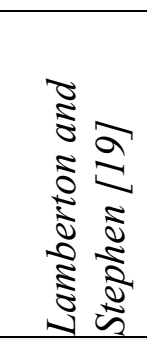 & 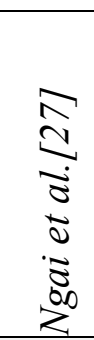 & 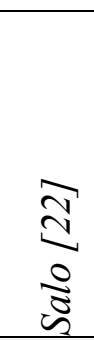 & 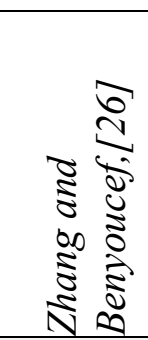 & 胥 \\
\hline $\begin{array}{l}\text { Social Media Practice } \\
\text { and Strategy }\end{array}$ & $\mathbf{X}$ & & & $\mathbf{X}$ & $\mathbf{X}$ & $\mathrm{X}$ & $\bar{X}$ & $X$ & & $X$ & $\mathbf{X}$ & $\mathbf{X}$ & $\mathbf{X}$ & 10 \\
\hline $\begin{array}{l}\text { Social Media User } \\
\text { Behaviour }\end{array}$ & $\mathbf{X}$ & & $\mathbf{X}$ & $\mathbf{X}$ & $\mathbf{X}$ & $\mathbf{X}$ & & $\mathbf{X}$ & $\mathbf{X}$ & $\mathbf{X}$ & & & $\mathbf{X}$ & 9 \\
\hline $\begin{array}{l}\text { Social Media } \\
\text { Organizational } \\
\text { Context }\end{array}$ & $X$ & $\mathbf{X}$ & $\mathbf{X}$ & $\mathbf{X}$ & $\mathbf{X}$ & & $\mathbf{X}$ & & & $\mathbf{X}$ & $\mathbf{X}$ & $\mathbf{X}$ & & 9 \\
\hline $\begin{array}{l}\text { Theoretical } \\
\text { Considerations }\end{array}$ & $\mathbf{X}$ & $\mathbf{X}$ & & $\mathbf{X}$ & & $\mathbf{X}$ & & $\mathrm{X}$ & & $\mathbf{X}$ & & $\mathbf{X}$ & & 7 \\
\hline $\begin{array}{l}\text { Social Media } \\
\text { Research Approaches }\end{array}$ & $\mathbf{X}$ & $\mathbf{X}$ & & $\mathbf{X}$ & $\mathbf{X}$ & $\mathbf{X}$ & & $\mathrm{X}$ & & & & $\mathbf{X}$ & $\mathbf{X}$ & 8 \\
\hline $\begin{array}{l}\text { Social Media Privacy } \\
\text { and Security } \\
\text { Considerations }\end{array}$ & & & & & $X$ & & & & & $X$ & $X$ & & & 3 \\
\hline
\end{tabular}


Social media practice and strategy: Some authors focus on generic issues such as how companies use social media and how their social media marketing strategies differ [23, 32], whilst others point to the importance of improving understanding of the critical success factors associated with the implementation of social media $[24,28]$, whilst other authors propose more research into the impact of social media on firm performance [26]. Other SLRs also point to the importance of research into social media marketing evaluation [29, 33] Finally, Lamberton and Stephen [22] suggest that research is needed into the relationship between social media and other marketing channels, and on differentiation on the basis of customer segments.

Social media user behaviour: Several authors suggest that more research is needed with respect to the impact of social media on the decision-making processes around purchasing $[22,27]$ including impulse purchasing and how consumers move from one decision-making stage to another [24, 29]. Other authors suggest research on other aspects of social media use, such as user co-creation in social media marketing [28] and the impact of social media marketing on customer loyalty [24, 33]. Lamberton and Stephen [22] also suggest that it is necessary to gather a deeper understanding of why people use social media, and how it affects their lives. Some SLRs point to the need to understand the impact of different social media channels (e.g. Twitter, Facebook) on consumers $[32,33]$.

Social media organizational context: Only four of the SLRs comment on the organizational context in which any future research might be conducted. Abed et al [33], focusing on social media and e-commerce on a small to medium sized enterprise (SME), identified a number of aspects of the use of social media in SME's that would benefit from further research. These include: the link between SME characteristics and industry and the choice of social media marketing tools; the level of management support for SME's, and, 
the contribution of social media in SME's' internationalization. Other SLRs do not focus explicitly on a business sector but do suggest generic questions whose answers would be of value to businesses. Alves et al [32] suggest social media marketing in B2B value cocreation, whilst Baethge et al [26] sees a need for research into social commerce business models, mobile social commerce, social media and product type, small businesses, and channel strategies.

Social media privacy and security concerns: Somewhat surprisingly, only three SLRs called for more research into social media and privacy. Whilst, there is a case for suggesting that security and privacy are technical topics whose research does not lie in the realm of marketing and business, these are important concerns for organizations and consumers. Lamberton and Stephen [19] simply recognize that research is required into regulation and digital consumer privacy issues. Ngai et al [30] pose a useful generic research question: ' how does a firm apply technologies and train staff to manage the availability, sorting and filtering of data captured or used in social media to ensure the wise use of data and protect the privacy of all stakeholders (p. 41). Baethege et al [26] suggest the need for research into the extent to different security instruments and privacy protection strategies, promote user's positive perceptions of a social commerce platform, and are effective in fostering trust and engagement.

Theoretical considerations: Some SLRs comment on the need for theory to underpin research and offered recommendations as to how this might be achieved [25]. Abed et al [33] identifies the need to examine social media in SME's from a theoretical perspective, but does not develop this further. Other SLRs identify specific theories that could be applied in future research, such as co-creation of value [28, 32], Resource Based Theory [25], Service Dominant Logic ([28], mobile marketing theory [22] and Information Systems theories [24], 
Social media research approaches: Whilst seven of the articles made suggestions regarding the research approaches that should be used in future research, their commentary on this is succinct, and consensus is limited. Busalim et al [24] point to the need to develop the theoretical underpinning of social media marketing, and to use this to inform research questions. Some SLRs promote the need for more quantitative studies [26, 28, 33]. In this context, Baethge et al [26] suggest the need for developing understanding of big data methods to support the analysis of large social media data sets, whilst, consistent with this, Ketonen-Oksi et al [28] suggest that there is a need for more large-scale empirical research. On the other hand, other SLRs suggest that more qualitative work is needed, because the majority of the existing research is quantitative [32] and there is some support for further use of mixed methods research [28, 29].

\section{Discussion}

\subsection{SLR purpose}

The purposes for conducting the SLRs included in this study are diverse and offer insights into the purposes for which SLRs can be conducted. First, with the occasional exception (e.g. [26]) most authors see their audience for the SLR to be both researchers and practitioners. (e.g. $[22,24])$. This is consistent with the longstanding aspiration that research should inform both research and practice. However, whilst this aspiration is laudable, there are several challenges to be overcome before this can be achieved. Various authors have recognized both the benefits and the challenges of evidence-based practice [28, 29, 25, 31]. Such investigations are timely considering the increasing uptake of social media in modern marketing campaigns. Rowley's [20] suggestion that such practices will bridge the practicetheory divide is particularly appropriate within this respect. 
Table 2 makes an important contribution by identifying the espoused purposes of a range of SLRs within a specific field. Most authors are seeking to serve a range of objectives in their study, with some specifying a wider range than others. Lamberton and Stephen [22] and Busalim et al [24] state the widest range of objectives for their SLRs, whilst Abed [33] has the most limited number of purposes. The three most cited purposes are: making sense, helping researchers and practitioners, and identifying key issues. Somewhat surprisingly, the lowest two are developing a research agenda, and establishing rigour.

\subsection{SLR Processes}

Table 3 proposes a six-stage model of the processes in conducting an SLR, based on the articles selected for this review. As discussed below, in general this framework aligns well with the frameworks proposed by other SLR commentators. There is a general consensus that SLR's should have a number of specified steps $[12,13,16]$, but as is evident in Table 1, the steps are articulated differently in different models. Most authors suggest the need to plan and justify the review, represented in this article as step 1, which involves both formulating the objectives of the review and identifying research questions. Tranfield et al [12] then cluster a number of activities under the heading 'conducting the review', however, these do not readily align with Torroaco's [16] 'search and retrieve the appropriate literature(s)', or, the equivalent from this study: 'developing search strategies and identify relevant documents'. No prior authors specifically regard the formulation of exclusion criteria as a specific and separate step $[12,13]$. On the other hand, most are agreed that there is a need to analyse the final dataset $[12,13,16]$, and to develop a future research agenda $[10,12,13,16]$. On the other hand, no prior authors identify the development of a concept matrix and thematic analysis as a separate step. However, it is important to observe that the processes identified and summarised in Table 3 are based on an analysis of SLR practice, whilst other commentators offer good practice advice. 
One important stage has not received an explicit mention in previous studies, or in the SLRs reviewed in this article, is conducting a pilot study [34]. Such a pilot study should involve one or more preliminary searches to scope the terminology of the field, to gauge the extent of the knowledge base, and, most importantly, to identify any previous literature reviews in the field. The pilot study should lead to decisions regarding the scope of the search, in terms of, for example, topic, date range, search terms, and search engine. It may be that the SLRs studied did do this but omitted to report on this stage.

Ultimately, this study shows that there are considerable variations in the execution of SLRs, and, indeed, the extent to which the processes for conducting the review are executed. Whilst there is always a need for researchers to make judgements in their research, and the processes and approaches might legitimately vary because different authors have cited different purposes/objectives for their literature reviews, there remains considerable scope for further discussion leading to a consensus regarding both the purposes and processes of SLRs.

Earlier studies have also recognized the limitations of current practices. For example, Watson [17] criticizes the current approach to SLRs on the basis that the search process is inefficient and flawed. He proposes a system in which journals require authors to code the knowledge embedded in their articles, with the aid of a free open source word processor that stores text in an open document format. He also suggests that another way forward might be through text mining. Whilst the implementation of such approaches may take some time to develop and become mainstream, Watson's [17] article points to some of the limitations in the current approach to SLRs and should be considered alongside the findings from this research.

\section{Reflection and Recommendations}


This article has sought to demonstrate the extent to which undertaking an SLR is very different from developing a narrative literature review. Indeed, it should be evident that conducting such a review in an area in which the field has a significant literature base is a 'research project' in its own right, which demands resources and time as well as a specific suite of research expertise. From the perspective of an author, the downside of investing effort in an SLR is that not all research assessment and performance processes (in universities) will accord the same status to SLR articles as they might to an 'empirical' article. More positively, however, a well-crafted review on a topical theme has a very good chance of attracting a considerable number of citations, which builds the reputation and visibility of both the author(s) and the journal in which it is published. The other significant gain from taking time to focus on the past literature in a structured way is that the authors have a very clear picture of the research gaps in their field, often both in terms of topics and methodological traditions, on which to base their future research plans.

Switching to the wider perspective of the role of SLR's in advancing research and practice in social media marketing and the disciplines to which it contributes, management and information sciences, there is significant scope for enhancing SLR practice. A strong foundation of informative, authoritative and timely SLRs is essential in fields in which research and practice are developing rapidly. As a foundation for the consideration of SLR protocols, the following issues will need attention:

- Leadership and responsibility for the development of authoritative SLR protocols in management and information sciences. Leadership must be at organisational and not individual level, and lie with organisations that have responsibility for research and practice in the discipline area, such as professional bodies, and international research associations. 
- The evaluation of the applicability and value of different types of review $[4,35]$.

- Achieving relevance to practitioners through design of protocols, reporting and publication [36].

- The dynamism of the field, as evidenced by changing technologies, procedures, beliefs, communication channels, and operational principles [37]

- The ontological and epistemological idiosyncrasies of the field, including theoretical boundaries, the range of theoretical lenses adopted, the unit of analysis, the sources and levels of data collection, the study context, the definitions and operationalisation of constructs (including the extent of conceptual heterogeneity), and the research methods [38].

\section{Conclusion}

This article contributes to research by performing an overview of recent literature reviews in a specific area, social media marketing. The reviews vary in their specific focus, covering topics such as social media marketing, social commerce, social strategy, consumer behaviour in social commerce, and social media-based value creation and business models. They also take different perspectives on the relative importance of theory, research and practice. Nevertheless, whilst there are some commonalities in their approach to an SLR, and common themes emerging regarding future research agendas, there is also a surprising level of disparity in both of these areas. Leadership, supported by future research into the purpose, processes and contribution of SLRs would have the potential to develop good practice in the conduct of SLRs, and thereby improve the processes whereby researchers ground their research in previous knowledge and theory. 


\section{Declaration of conflicting interests}

The author(s) declare no potential conflicts of interest with respect to the research, authorship and/or publication of this article.

\section{Funding}

The author(s) received no financial support for the research, authorship and/or publication of this article.

\section{References}

[1] Tranfield D and Starkey K. The nature, social organisation and promotion of management research: towards policy. British Journal of Management, 1998; 9: 341353.

[2] McGaghie W C. Varieties of integrative scholarship: Why rules of evidence, criteria, and standards matter. Academic Medicine 2015; 3: 294-302.

[3] Rowley J and Slack F. Conducting a Literature Review. Management Research News 2004; 27: 31-39.

[4] Wulff J N. A systematic assessment of empirical research on foreign entry mode. European Journal of Marketing 2016; 50: 942-972.

[5] Aguinis H, Ramani, R S and Alabduljader, N. What you see is what you get? Enhancing methodological transparency in management research. Academy of Management Annals 2018; 12: 1-28.

[6] Cook D J, Greengold N L, Ellrodt A G and Weingarten S R. The relation between systematic reviews and practice guidelines. Annals of Internal Medicine 1997; 127: 
210-216.

[7] Littel J, Corcoran J and Pillai, V.) Systematic Literature Reviews and Meta-analysis. New York: Oxford University Press, 2008.

[8] Webster J and Watson R T Analyzing the past to prepare for the future: writing a review. MIS Quarterly 2002; 26: 8-23.

[9] Moher D, Liberati, A, Tetzlaff J, Altman D G. Preferred reporting items for systematic reviews and meta-analyses: The PRISMA Statement. PloS Medicine 2009; 6: 7 e1000097

[10] Crossan M M and Apaydin M.. A multi-dimensional framework of organizational innovation: a systematic review of the literature. Journal of Management Studies 2010; 47: 1154-1191.

[11] Tranfield D, Denyer D and Smart P. Towards a methodology for developing evidence-informed management knowledge by means of systematic review. British Journal of Management 2003; 14: 207-222.

[12] [C] Harris J L, et al. Cochrane Qualitative and Implementation Methods Group guidance series - paper 2: methods for question formulation, searching, and protocol development for qualitative evidence synthesis. Journal of Clinical Epidemiology 2018; 97: 39-48.

[13] Fisch C and Block J. Six tips for your (systematic) literature review in business and management research. Management Review Quarterly 2018; 68: 103-106.

[14] Boell S K and Cecez-Kecmanovic D. On being 'systematic' in literature reviews. Journal of Information Technology 2015; 30: 161-173.

[15] Denyer D and Tranfield D. Using qualitative research synthesis to build a 
actionable knowledge base. Management Decision 2006; 44: 213-227.

[16] Torraco R J. Writing integrative literature reviews: guidelines and examples. Human Resource Development Review 2005; 4: 356-367.

[17] Watson R T. Beyond being systematic in literature reviews in IS. Journal of Information Technology 2015; 30: 185-187.

[18] Denyer D, Tranfield D and Van Aken J E Developing design propositions through research synthesis. Organization Studies 2008; 29:393-413.

[19] Alalwan A A, Rana N P, Dwivedi Y, and Algharabat R.). Social media in marketing: a review and analysis of the existing literature. Telematics and Informatics, 2017; 34: 1177-1190.

[20] Rowley J. Evidence-based marketing: a perspective on the "practice-theory divide. International Journal of Market Research (2012); 54: 521-541.

[21] Denyer D and Tranfield D). Producing a systematic review. In:Buchanan, D. (ed) The Sage Handbook of Organizational Research Methods. Thousand Oaks, Sage (2009pp. 671-689.

[22] Lamberton C and Stephen A T. A thematic exploration of digital, SM, and mobile marketing: research evolution from 2000 to 2015 and an agenda for future inquiry. Journal of Marketing 2016; 80: 146-172.

[23] Effing R and Spil T A M. The social strategy cone: Towards a framework for evaluating social media strategies. International Journal of Information Management 2016; 36: 1-8.

[24] Busalim A H, Razak A and Hussin C. Understanding social commerce: A systematic literature review and directions for further research. International 
Journal of Information Management 2016; 36: 1075-1088.

[25] Salo J. Social media research in the industrial marketing field: Review of literature. Industrial Marketing Management 2017; 66: 115-129.

[26] Baethge C, Klier J and Klier M. Social commerce-state-of-the-art and future research directions. Electronic Markets 2016; 26: 1-22.

[27] King R A, Racherla P and Bush V D. What we know and don't know about online word-of-mouth: A review and synthesis of the literature. Journal of Interactive Marketing 2014; 28: 167-183.

[28] Ketonen-Oksi S, Jussila J J and Kärkkäinen H Social media based value creation and business models. Industrial Management and Data Systems 2016; 116: 1820- 1838.

[29] Zhang K Z and Benyoucef M. Consumer behavior in social commerce: A literature review. Decision Support Systems 2016; 86: 95-108.

[30] Ngai E W T, Tao S S C and Moon K K L. Social media research: Theories, constructs, and conceptual frameworks. International Journal of Information Management 2015; 35: 33-44.

[31] Ahmed Y A and Zakaria N H. Social media for knowledge sharing: a systematic literature review. Telematics and Informatics 2018. In Press.

[32] Alves H, Fernandes C and Raposo M. Social media marketing: A literature review and implications. Psychology and Marketing 2016; 33: 1029-1038.

[33] Abed S S, Dwivedi Y K and Williams M D). Social media as a bridge to e- commerce adoption in SMEs: A systematic literature review. The Marketing Review 2015; 15: 3957. 
[34] Bryman A and Bell E. Business Research Methods. New York. Oxford University Press, 2010.

[35] Pare G, Trudel M-C, Jaana M, Kitsiou, S. Synthesizing information systems knowledge: A typology of literature reviews. Information \& Management 2015; 52: 183199.

[36] Adams R J, Smart P, Huff, A S. Shades of grey: Guidelines for working with the grey literature in systematic reviews for management and organizational studies. International Journal of Management Reviews 2017; 19: 432-454.

[37] Durach C, Kembro J, Wieland A. A new paradigm for systematic literature reviews in supply chain management. Journal of Supply Chain Management 2017; 53 (4): 67-85. 